\title{
Resonance frequency analysis of laser optical fiber based on microcantilever
}

\author{
Mohanad Aljanabi \\ Electrical Power Techniques Engineering, Technical College/AL-Mausaib, Al-Furat Al-Awsat Technical University, Iraq
}

\begin{tabular}{l}
\hline \hline Article Info \\
\hline Article history: \\
Received Dec 30, 2018 \\
Revised Mar 28, 2019 \\
Accepted Apr 8, 2019 \\
\hline
\end{tabular}

\section{Keywords:}

Biosensor

Laser

Microcantilever

Optical fiber

Resonant frequency

\begin{abstract}
The normal frequency of smart beams was originated utilizing FEM [Ansys and Comsol] code for first five modes by varying the position of actuator from the fixed end of the structure, and it has a suitable arrangement with analytically found the standard frequency. This paper includes learning a resonance frequency analysis of laser optical fiber based on microcantilever of designing magnetic actuator using Ansys and Comsol simulation. The design of optical fiber includes Nickel cantilever, two magnets and one coil that apply to force on the cantilever. After the current flows in the coil domain, the shape of microcantilever will be deformed. It will move to $\mathrm{z}$ - direction that depends on the force direction. Two methods including, Comsol Multiphysics, Ansys and analytical equations have been utilized to calculate the resonance frequency, current and force values. The simulation results include calculating the current (magnetic current density) and effects of the magnetic field of the coil on the cantilever (force calculation). Utilizing this method is to limit faults (errors) of optical fiber laser between transmitter and receiver system (detection system) for any time of cutting coil when the signal of a laser passes through the coil. In conculsions, resonant frequency (f_n) tuning using cantilivier presented in the resrach have larger variable range by using simulations. However, the adjusting of the system and changing the deminsions.Resolutions to this problematic contain tuning the modes of resonant frequency to produce by cantilivier with 2-magnets and coil when the signal pass from laser source. Based on these simulations and characterization results, the proposed assembly can be a good applicant for evolving a low price, high material platform for many biological, laser optical fiber, communication, machine learning, biosensors and biomedical applications.
\end{abstract}

Copyright (C) 2019 Institute of Advanced Engineering and Science. All rights reserved.

\section{Corresponding Author:}

Mohanad Aljanabi,

Electrical Power Techniques Engineering,

Technical College /AL- Mausaib, Al-Furat Al-Awsat Technical University,

Najaf, Iraq.

Tel: 009647735401438

Email: aljanabimohanad@gmail.com

\section{INTRODUCTION}

Flexible structures frequently have low rigidity and slight material damping ratio. The excitation may lead to critical large amplitude vibration and long settling time. These can result in fatigue, instability and poor operation of the structures. Vibration control of flexible structures is a significant problem in various science applications, particularly for the precise operation parameters in aerospace systems, satellites, a magnetic actuator, flexible manipulators, laser optical fiber, modes of frequency resonance with transmitter and receiver, transducer, biosensor of nanoantennas, etc. [1-3]. 
Different kinds of cantilevers which are made of various materials have been advanced as transducers utilized in biosensors [4-6]. Regarding actuating and sensing techniques, all the cantilevers can be divided into two categories: passive and active. The passive cantilevers, such as silicon-based cantilevers, need a separated system to actuate the device and typically utilize a separated optical system to measure/monitor the vibration of the device. The active cantilevers, for example, piezoelectric-based cantilevers, can be easily motivated by simply applying a driving field, an electric field(E) in the piezoelectric, and the vibration behaviour of the active cantilever can be directly sensed/monitored, by measuring impedance in the piezoelectric [7-9]. Because of the easiness and availability of the micro/nanofabrication method, silicon-based cantilevers are much more extensively investigated than others. Additionally, silicon-based cantilevers show a higher $\mathrm{Q}$ value than piezoelectric-based cantilevers and laser optical fiber [10].

The objective of this paper is to design a magnetic actuator that has microcantilever, two magnets and coil by using a Comsol and Ansys simulation. The parts of magnetic actuator that are fifty turns coil, $\mathrm{Ni}$ cantilever and two magnets. The voltage is applied to the coil to produce current flows when current flows through the coil magnetic field occurs. After that magnetic field applies force on Ni (ferromagnetic material) microcantilever. This force caused to deform the cantilevers [11], microcantilevers that use magnetic actuation to utilize as mass sensors for bio-applications. The microcantilevers are made of electroplated cobalt-nickel that has specific properties these are low conductivity and high saturation magnetisation. Instead, the microcantilevers are stimulated by applying electromagnetic fields (E, H) [12]. Optical fiber sensors are classified depend on the position, operation principle and applications to three groups. In the first group, optical fibers are divided as an extrinsic or intrinsic. In an extrinsic, the fiber is utilized to carry light to and from an external optical device where the sensing takes place. Also, fiber is work as a leader to deliver light to the sensing position. Despite the fact in an intrinsic case, the physical properties of the fibers are focusing to change. These changes are works on the optical fiber to change approximately of the light distinguishing as shown in Figure 1.

The main parts of the micro magnetic actuator should consist of a substrate, a coil, magnetic physical, and a portable membrane [13]. The working principle of the device in literature is that the current is useful to the coil, then a magnetic field is produced which interacts with the magnetic field of the permanent magnet to produce a force. This force acts on the membrane thereby causing it to deflect. The deflection primarily depends on the magnetic force generated by the coil and the magnet, and the restoring force of the membrane $[14,15]$.

Electromagnetic actuators characteristically can be worked at substantially smaller voltages but might require larger driving currents. Optical sensors typically work depend on emitting light to the surface of the cantilever with a laser and analysing the location of reflected light to measure the beam movement [16]. Figure 2 shows the optical detection for cantilever technique.

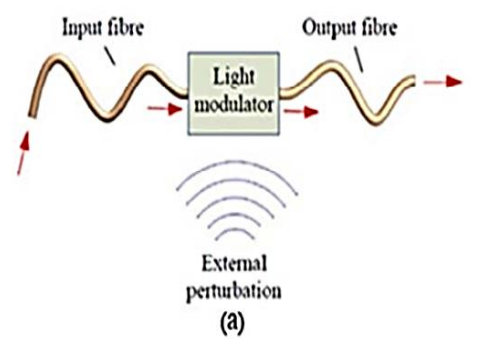

Figure 1. Extrinsic and intrinsic fiber optic sensors [13]

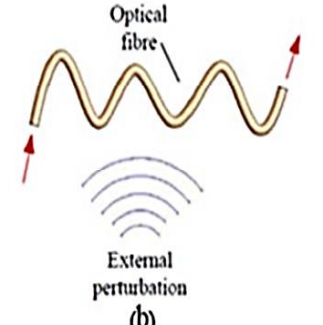

(b)

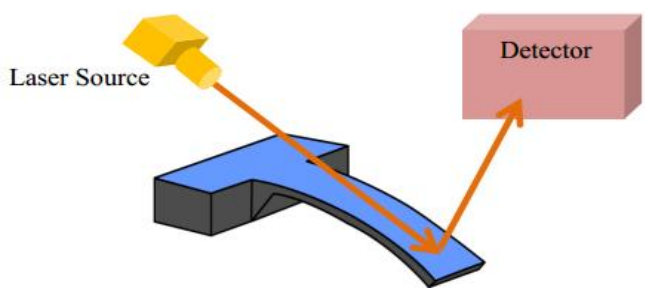

Figure 2. Optical detection for cantilevers [16]

However, several studies projected utilizing multiplexing technique to decrease the number of sources and detectors. For instance, in 2012 a set advanced a system for monitoring eight cantilevers [17]. This format involves of 8 Vertical-cavity surface-emitting laser and one photodetector. Also, it is probable to use one CCD image screen as a detector for wholly biosensors. Yue et al. utilise one collimated beam as a light source for 100 of cantilevers and distinguish their difference with a CCD plane [17]. In addition, having high sensitivity and good determination, another benefit of optical laser detection is the ease of the fabrication process for sensors which are going to be utilised with an optical laser setup. For any cantilever sensor, there are two properties of the frequency response that are of central importance: the position of the resonance frequency $\left(f_{n}\right)$ and the quality factor (Q-factor), given by $Q=\Delta f / f 0$, where $\Delta f$ is the FWHM of the 
resonance peak $[18,19]$. But, since detection in these sensors will be completed depend on light's reflection from a surface, it is significant to have a uniform surface to avoid light scattering [20].

Now that the magnetic flux density around the magnets is characterized, dynamic magnetic analysis can be performed to determine how much voltage is expected to be generated with the chosen winding configuration. The voltage generated by a coil is the derivative of the flux linkage with respect to time [21]. To narrow down the scope of the analysis, the general configuration of the magnets and the coils are determined first. The simplest way to increase the change in magnetic flux is to use a strong magnet. Another way to achieve the same is to use two different magnets that are attached side-by-side with different dimensions [22]. It is evident that electromagnetic forces have the potential to generate large deflections [22]. Utmost of the magnetic microactuators advanced until now are dependent on the variable reluctance principle and use soft magnetic materials [23]. Nevertheless, hard magnetic materials are deemed as desirable actuator components because of their favourable scaling and the resulting potential for larger forces and larger deflections, applicable to milli-scale actuators. Permanent magnet microactuators have been previously confirmed either using hybrid assembled, commercially available permanent magnets [24, 25]. Development of bio-sensing applications led to in a great interest in cantilever-based micro-resonators. Dynamic mode sensing is typically favoured as it makes available a higher sensitivity in mass measurements compared to static deflection measurements [24].

\section{METHODS}

Fabrication sequence of magnetic micro-actuator is shown in Figure 3 [25]. This process combine ordinary micromachining methods "sacrificial layer methods". The process stages are electroplating, screen printing and lamination. Micro-fabrication of the Micro-cantilevers is depended on two lithography stages: Electroplating stage and Sacrificial layer etching stage, as shown in Figure 4.

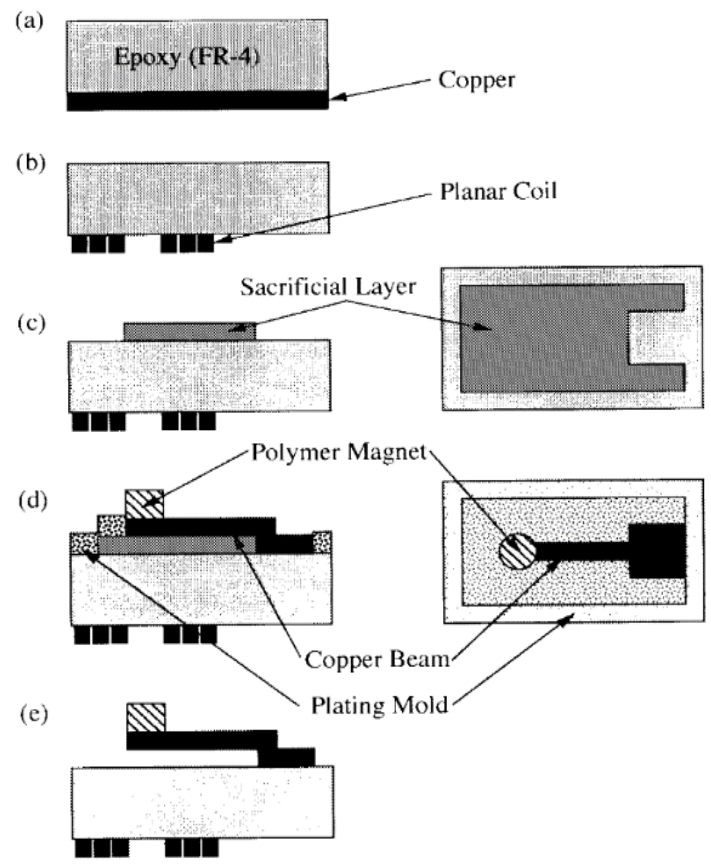

Figure 3. Stages of the fabrication process of the magnetic actuator
1

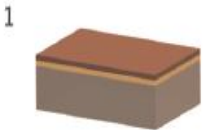

4
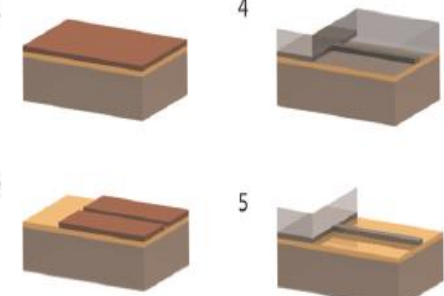

5

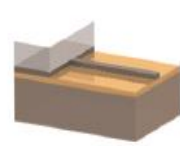

$\square$ Copper

- Silicon

$\square \mathrm{CONi}$

$\square$ SU-8

$\square$ Exposed SU-8

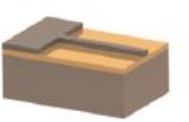

6

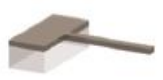

Figure 4. Steps of fabrication of the cobalt-nickel micro-resonators

The membrane was fabricated by $\mathrm{KOH}$ etching of a silicon wafer [26]: LPCVD deposition of silicon nitride; Spin on, expose and develop resistance; Plasma etches silicon nitride; Eliminate resist and Timed $\mathrm{KOH}$ etching of silicon wafer to produce membranes, as shown in Figure 5. 

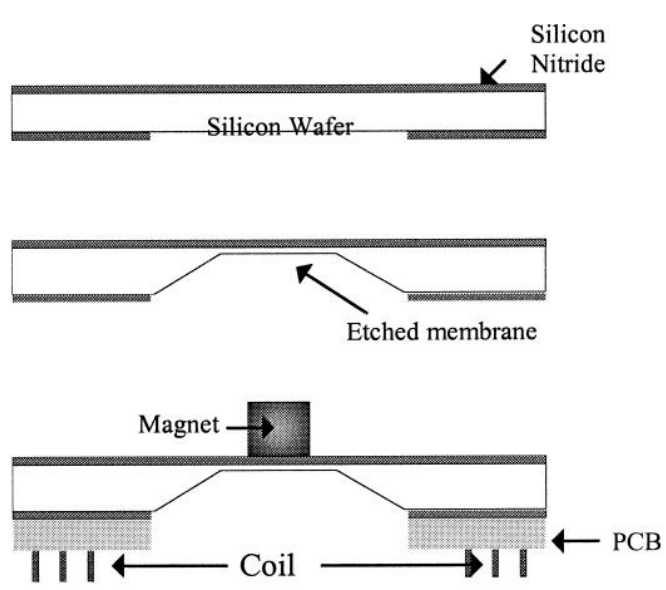

Figure 5. The membrane was fabricated by $\mathrm{KOH}$ etching of a silicon wafer with coil

Advantages of the magnetic actuator [23]: 1) Electromagnetic actuation has the benefit of contactless movement; 2) The possibility of dropping power consumption in magnetic actuators can be done by utilizing high ratio coils varieties electromagnetics an good-looking optimal of driving opinion; and 3) Necessary distinguishing of having the capacity to produce large forces which agrees on the approach of devices with large deflection.

In this paper, the Comsol Multiphysics program has been utilized to get benefit from the tool and its application areas to design microcantilever. The literature of the magnetic actuator is studied to understand its basics. This research has culminated with designing of the magnetic actuator. At the same time, the magnetic actuator was drawn by using Comsol tool to calculate current, magnetic field and force of coil on the cantilever [26].

1. First, the Magnetic actuator as Figure 6 and Figure 7 is created and drawn by using Comsol tool.

2. Specified element type of device and material properties; Young's Modulus and Poisson Ratio, relative permeability, relative permittivity, and conductivity for Ni cantilever, as Table 1 and Table 2 .

3. Moreover, we also entered other material properties for coil and magnets.

Table 1. First part of search with (for Figure 6)

\begin{tabular}{llllll}
\hline \multicolumn{1}{c}{ Elements } & Young's Modulus (Pa) & Poisson Ratio & Relative Permeability & Relative Permittivity & Conductivity (S/m) \\
\hline Air (all system) & - & - & 1 & 1 & - \\
Ni cantilever & $219 \mathrm{e} 9$ & 0.31 & 100 & 1 & $13.8 \mathrm{e} 6$ \\
Magnet & & & 5000 & 1 & sigma(T[1/K]) [S/m] \\
Coil & & 1 & 1 & $3.7 \mathrm{e} 7$ \\
\hline
\end{tabular}

Table 2. Second part of search with (for Figure 7)

\begin{tabular}{llllll}
\hline Elements & Young's Modulus (Pa) & Poisson Ratio & Relative Permeability & Relative Permittivity & Conductivity (S/m) \\
\hline Air (all system) & - & - & 1 & 1 & - \\
Ni cantilever & $219 \mathrm{e} 9$ & 0.31 & 100 & 1 & $13.8 \mathrm{e} 6$ \\
Magnet & & & 5000 & 1 & sigma(T[1/K]) $[\mathrm{S} / \mathrm{m}]$ \\
Coil & & 1 & 1 & $6 \mathrm{e} 7$ \\
\hline
\end{tabular}

The basic equations of the magnetic and electric field;

$$
\begin{aligned}
& \nabla \cdot \mathrm{J}=0 \\
& \nabla \times H=J \\
& J=\sigma E+J_{e} \\
& E=-\nabla V \\
& B=\nabla \times A
\end{aligned}
$$


The basic equations of Ampere's Law and Current Conservation;

$$
\begin{aligned}
& \nabla \times\left(\mu_{0}{ }^{-1} \mu_{\mathrm{r}}{ }^{-1} \mathrm{~B}\right)-\sigma \mathrm{v} \times \mathrm{B}=\mathrm{J}_{\mathrm{e}} \\
& \mathrm{B}=\nabla \times \mathrm{A} \\
& \nabla \cdot \mathrm{J}=0 \\
& \mathrm{D}=\epsilon_{0} \epsilon_{\mathrm{r}} \mathrm{E}
\end{aligned}
$$

The equations of Magnetic and Electric Insulation;

$$
\begin{aligned}
& \mathrm{n} \times \mathrm{A}=0 \\
& \mathrm{n} \cdot \mathrm{J}=0
\end{aligned}
$$

Force Calculation;

$$
\begin{aligned}
& \mathrm{F}=\int_{\partial \Omega} \mathrm{nTd} S \\
& \tau=\int_{\partial \Omega}\left(r-r_{0}\right) \times(n T) d S \\
& \tau=\frac{r_{a \times}}{\left|r_{a \times}\right|} \cdot \tau
\end{aligned}
$$

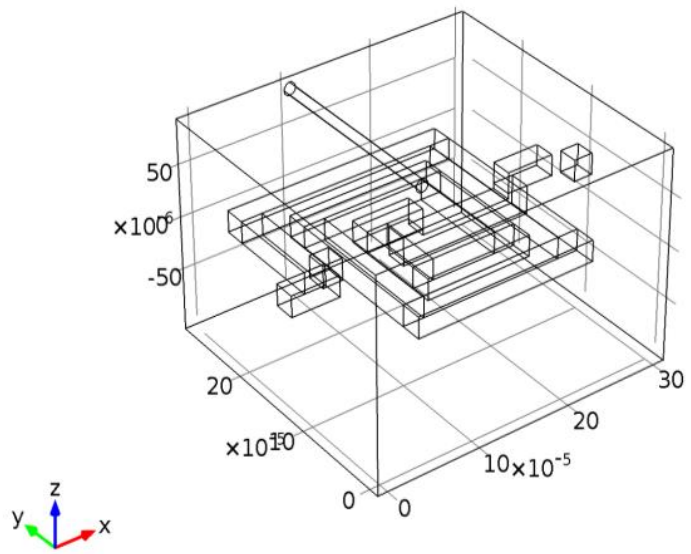

Figure 6. The structure of magnetic actuator (first part)

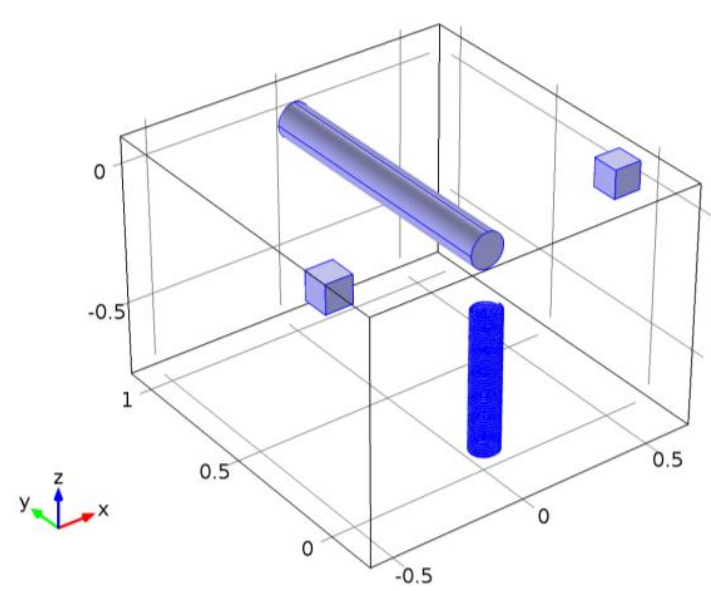

Figure 7. Magnetic actuator with $\mathrm{N}=50$ turn coil (second part)

The basic equations for the magnetic field;

$$
\begin{aligned}
& \mathrm{B}=\nabla \times \mathrm{A} \\
& \nabla \times \mathrm{H}=\mathrm{J}_{\mathrm{e}}
\end{aligned}
$$

The basic equations of Ampere's Law;

$$
\begin{aligned}
& \nabla \times\left(\mu_{0}{ }^{-1} \mu_{\mathrm{r}}{ }^{-1} \mathrm{~B}\right)-\sigma \mathrm{V} \times \mathrm{B}=\mathrm{J}_{\mathrm{e}} \\
& \mathrm{B}=\nabla \times \mathrm{A}
\end{aligned}
$$


The equations of Magnetic Insulation;

$$
\begin{aligned}
& \mathrm{n} \times \mathrm{A}=0 \\
& \nabla \times\left(\mu_{0}^{-1} \mu_{\mathrm{r}}^{-1} \mathrm{~B}\right)-\sigma \mathrm{v} \times \mathrm{B}=\mathrm{J}_{\mathrm{e}} \\
& \mathrm{B}=\nabla \times \mathrm{A} \\
& \mathrm{J}_{\mathrm{e}}=\frac{\mathrm{N}\left(\mathrm{V}_{\text {coil }}+\mathrm{V}_{\text {ind }}\right)}{\mathrm{AR}_{\text {coil }}} \mathrm{e}_{\text {coil }}
\end{aligned}
$$

The Comsol simulation will be compared with an analytical solution to verify that the same results can be achieved. The magnetic force, $\mathrm{F}(\mathrm{N})$, on the microresonator based on the magnetisation of the CoNi beam, $\mathrm{M}(\mathrm{A} / \mathrm{m})$, and magnetic field gradient produced by the actuation coil [27];

$$
\mathrm{F}=\mu_{0} \mathrm{v}(\mathrm{M} \dot{\times} \nabla) \mathrm{H}
$$

where $\mu_{0}\left(\mathrm{~N} / \mathrm{A}^{2}\right)$ is the permeability of free space, $\mathrm{n}\left(\mathrm{m}^{3}\right)$ is the volume of the micro resonator, $\nabla$ is the gradient operator, and $\mathrm{H}(\mathrm{A} / \mathrm{m})$ is the useful magnetic field.

\section{RESULTS AND DISCUSSIONS}

\subsection{Simulation results}

A Magnetic flux density norm using Ansys simulation, the 3D Plot Group 2 using Comsol simulation and the 3D Plot Group 3 Comsol simulation are shown as Figure 8, Figure 9 and Figure 10, respectively.

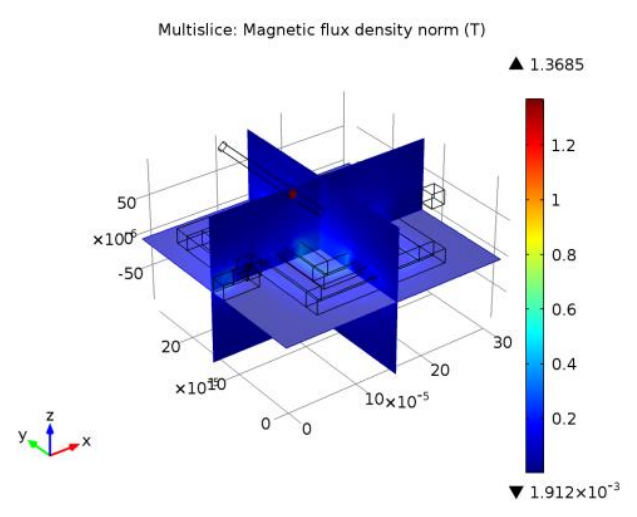

Figure 8. Multiline: Magnetic flux density norm (T)

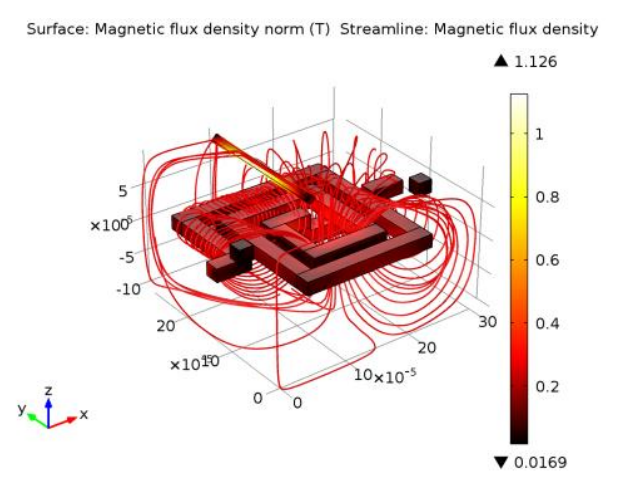

Figure 9. Surface of the Magnetic flux density standard (T) Streamline: Magnetic flux density

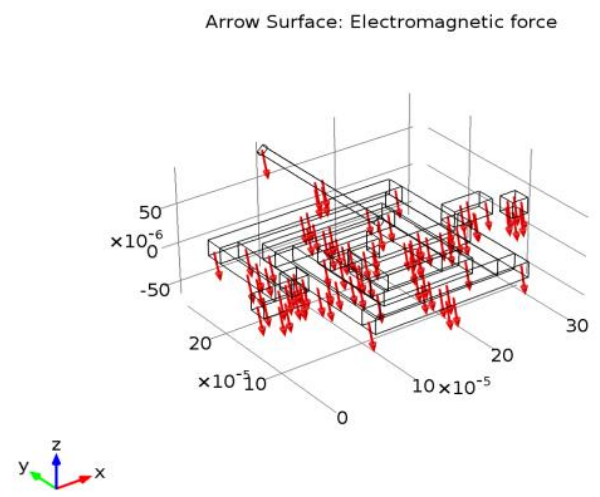

Figure 10. Arrow surface the electromagnetic force 
The magnetic actuation of the system contains an electro-coil, two permanent magnets for magnetisation of the cantilevers and a high-current coil. The first step is to design the model in the 3D figure as shown in Figure 11, the two magnets have the same size and shape, and they are parallel with a distance between them $24 \mathrm{e}-3 \mathrm{~m}$ and the dimensions of each other are $(3,15,11) \mathrm{e}-3 \mathrm{~m}$. The coil is positioned in the centre of the gap between magnets, the number of turns is 40 , and the thickness of the wire is $0.127 \mathrm{~mm}$, the outer radius of the coil is $10 \mathrm{~mm}$ so, the inner radius is $9.746 \mathrm{~mm}$. Figure 12 shows the cross-section of the coil. The magnetic actuation of were placed in parallel to each other in such a way to form a DC magnetic field with magnetic lines $(\mathrm{H})$ in the direction of the cantilevers. $\mathrm{H}$ makes magnetic dipole moments in the nickel cantilever along the DC field lines. After the cantilever is excited by the AC magnetic field formed by the electro-coil, the dipoles try to align themselves with the AC field vibrating the cantilever at the frequency of the AC magnetic field. The electro-coil was optimised to create maximum AC magnetic field over the cantilevers for the maximum current that can be obtained from the coil amplifier. We study the effects between two magnets and coil on the cylinder as a cantilever, the principle of the work of this study that when a current applied on coil, a magnetic field generated which studies with the magnetic field distribution $(\mathrm{H})$ of the permanent magnet to produce a force, this force acts on the cantilever so, the cantilever will vibrate with a resonance frequency.
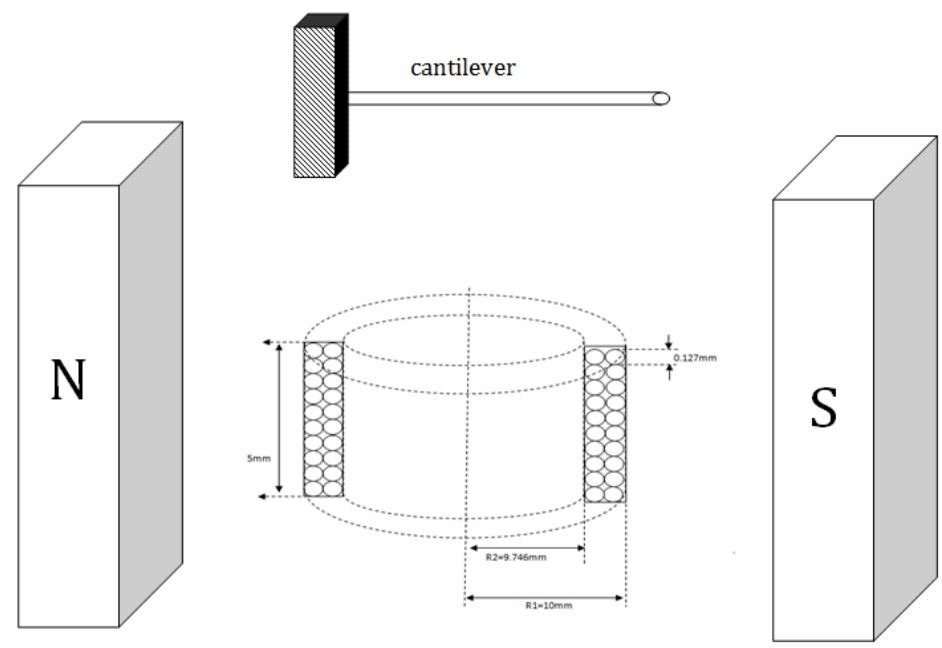

Figure 11. Schematic view of the two magnetic and coil with vibration of cantilever

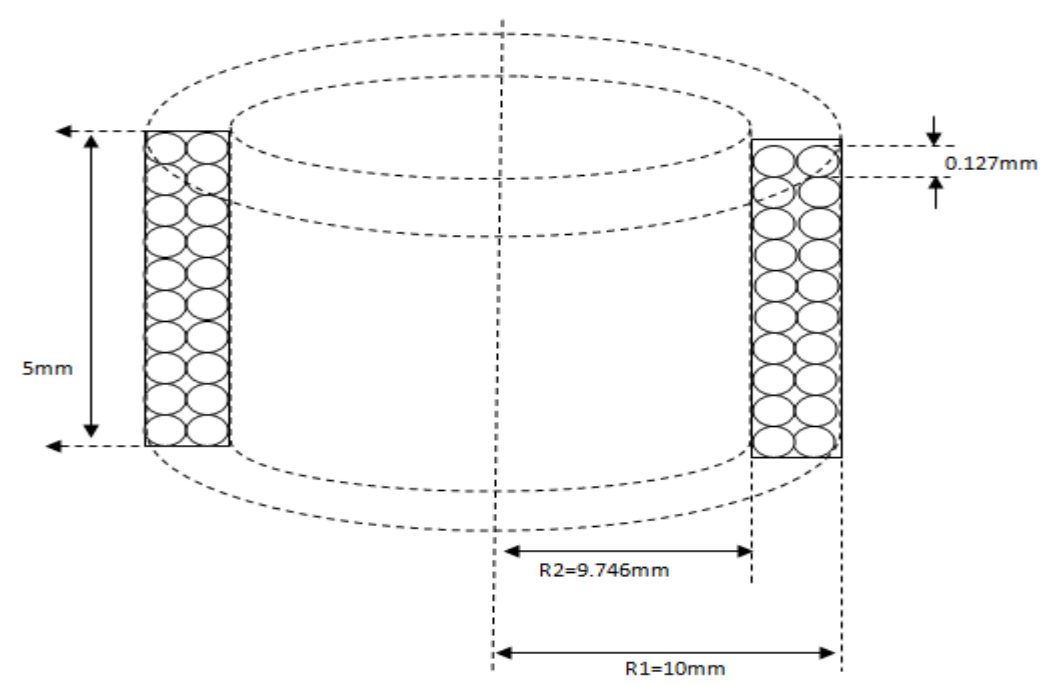

Figure 12. The cross section of the coil used 


\subsection{Analytical solution} solution;

When we compared the second part of the project simulation with the second part analytical

$$
\begin{aligned}
& B=\mu_{0}\left(\frac{N}{L}\right) I \\
& F B=I l B
\end{aligned}
$$

Table 3 shows different values of frequency calculated from the equation in different length from (1-5) $\mathrm{mm}$ with the values of frequency in Comsol simulation.

$$
f=\frac{3.52}{2 \pi l^{2}} \sqrt{\frac{E I}{\rho A}}
$$

Where $A$ is the area of the cross section of the cylinder (silica glass material), $\mathrm{d}=125^{*} 10^{-6} \mathrm{~m}, \mathrm{E}=73.1 * 10^{9}$ $\mathrm{Pa}, \rho=2203\left(\mathrm{Kg} \backslash \mathrm{m}^{3}\right)$ : -

$$
I=\frac{\pi d^{4}}{64}
$$

by substitution (2) in (1), we get

$$
f=\frac{3.52 d}{8 \pi l^{2}} \sqrt{\frac{E}{\rho}}
$$

Table 3. Parameters of analytical solution

\begin{tabular}{cc}
\hline Parameter & Value \\
\hline$\mu_{0}$ & $4 \pi 10^{-7}$ \\
$\mathrm{I}(\mathrm{A})$ & 1.9 \\
$\mathrm{~L}(\mathrm{~m})$ & $0.5 \times 10^{-3}$ \\
$\mathrm{~N}($ turns $)$ & 50 \\
$\mathrm{~B}$ & $\left(\mu_{0} N I\right) / L$ \\
$\mathrm{~F}$ & $I l \times B$ \\
$\mathrm{~F}(\mathrm{~N})$ & 0.0454 \\
Lorentz force $\left(\mathrm{N} / \mathrm{m}^{\wedge} 3\right)$ & $0.3632 \mathrm{e} 9$ \\
\hline
\end{tabular}

From Table 4, we saw that the values of analytical frequency for lower mode vibration is approximate to the first eigenvalue in Comsol also the frequency decreases as the length of the cylinder increases. Table 5 shows different values of frequency calculated from the equation for the mode of vibration $\left(\mathrm{m}_{1}, \mathrm{~m}_{2}\right.$ and $\left.\mathrm{m}_{3}\right)$ in different length from (1-5) $\mathrm{mm}$ with the values of frequency in Comsol simulation.

Table 4. Different values of frequency calculated with various length from (1-5) mm

\begin{tabular}{ccc}
\hline $\mathrm{L}(\mathrm{mm})$ & F1(Hz) Equation & F1(Hz) Comsol \\
\hline 1 & $1.0039944 \mathrm{e} 5$ & $1.00076 \mathrm{e} 5$ \\
2 & 25224.61138 & 25153.2307 \\
3 & 11210.938 & 11192.0518 \\
4 & 6306.152 & 6254.9795 \\
5 & 4035.9378 & 3918.83955 \\
\hline
\end{tabular}

$$
\begin{gathered}
E=\frac{64 \pi^{2} \rho l^{4} f_{n}^{2}}{m_{n}^{4} d^{2}} \\
f_{n}=\frac{m_{n}^{2} d}{8 \pi l^{2}} \sqrt{E / \rho}
\end{gathered}
$$


Table 5. Different values of frequency for mode of vibration $\left(\mathrm{m}_{1}, \mathrm{~m}_{2}\right.$ and $\left.\mathrm{m}_{3}\right)$ in different length from (1-5) $\mathrm{mm}$

\begin{tabular}{|c|c|c|c|c|c|c|}
\hline $\mathrm{L}(\mathrm{mm})$ & $\begin{array}{c}\mathrm{F}_{1}(\mathrm{~Hz}) \\
\text { Equation }\end{array}$ & $\begin{array}{l}\mathrm{F}_{1}(\mathrm{~Hz}) \\
\text { Comsol }\end{array}$ & $\begin{array}{c}\mathrm{F}_{2}(\mathrm{~Hz}) \\
\text { Equation }\end{array}$ & $\mathrm{F}_{2}(\mathrm{~Hz})$ Comsol & $\begin{array}{c}\mathrm{F}_{3}(\mathrm{~Hz}) \\
\text { Equation }\end{array}$ & $\begin{array}{c}\mathrm{F}_{3}(\mathrm{~Hz}) \\
\text { Comsol }\end{array}$ \\
\hline 1 & $1.008994 \mathrm{e} 5$ & $1.000763 \mathrm{e} 5$ & $6.41315 \mathrm{e} 5$ & $5.989104 \mathrm{e} 5$ & $17.678104 \mathrm{e} 5$ & $14.41526 \mathrm{e} 5$ \\
\hline 2 & 25224.617 & 25153.2307 & $1.603288 \mathrm{e} 5$ & $1.557336 \mathrm{e} 5$ & $4.41952602 \mathrm{e} 5$ & $4.279887 \mathrm{e} 5$ \\
\hline 3 & 11210.938 & 11192.0518 & 71257.26 & 69762.978 & $1.9642337 \mathrm{e} 5$ & $1.93684 \mathrm{e} 5$ \\
\hline 4 & 6306.152 & 6255.3218 & 40082.208 & 39177.8604 & $1.1048815 \mathrm{e} 5$ & $1.09385 \mathrm{e} 5$ \\
\hline 5 & 4035.9378 & 3933.1594 & 25652.613 & 24688.061 & 70712.416 & 68754.4271 \\
\hline
\end{tabular}

For analytical calculations, vibration mode divided by $2 \pi$ has been employed. Assume the cylinder is made of Silica glass in cantilever with $\mathrm{d}=125^{*} 10^{-6} \mathrm{~m}, \mathrm{E}=73.1 * 10^{9} \mathrm{~Pa}, \rho=2203\left(\mathrm{Kg}_{\mathrm{gm}}{ }^{3}\right), \sigma=0.17 \quad \mathrm{~m}_{1}=1.875$, $\mathrm{m}_{2}=4.730041, \mathrm{~m}_{3}=7.853205$.

\section{CONCLUSION}

The techniques of tuning the resonant frequency include changing the dimensions of the cantilever and 2-magnets and coil depending on the changing sizes, materials of the cantilever, source apply for this system (laser, IR) or any source working and matching between the structure of this parts. Therefore; it was concluded that applying loads to a cantilever is the best method to tune the resonant frequency of the vibration based is compared to applying compressive methods. Results from many tests which use cantilever platforms have shown that these sensors can demonstrate good sensitivity and high resolution. Moreover, for smart materials will control the design in the relationships of decreasing the vibrations amplitude and frequency to advance the efficiency of the designer in the new applications. Moreover, we study cantilevers dimensions with their effect on resonance frequency $\left(f_{n}\right)$. Unsing this technique for limit faults (errors) of optical fiber laser between transmitter and receiver system (detection system) for any time of cutting coil when the signal of laser pass through the coil.

This technique is suitable for low-cost multiplexed portable optical fibre laser to get the right new positions of detection. In our future studies, we will progress the detection accuracy by a rise the turns of the coil and improve the data by increasing the values of resonant frequency. The sensor technology confirmed is significant as it is scalable to larger arrays for simultaneous and real-time monitoring of various biological and chemical sensors.

\section{REFERENCES}

[1] Lavrik, N. V.; Sepaniak, M. J.; Datskos, P. G.,"Cantilever transducers as a platform for chemical and biological sensors," Review of Scientific Instruments, 75 (7), 2229-2253, 2014.

[2] Jalal Naghar, Otman Aghzout, Azzeddin Naghar, "Design Study of a Miniaturized Multi-layered Antenna-inpackage for 2.4 GHZ Wireless Communication," International Journal of Electrical and Computer Engineering (IJECE), Vol.8, No.5, pp.3627 3635, October 2018, DOI:10.11591/ijece.v8i5.pp3627-3635

[3] Ziegler, C. "Cantilever-based biosensors. Analytical and Bioanalytical Chemistry," 379 (7-8), 946-959. Sensors (2017), 7 2941, 2004.

[4] Yi, J. W.; Shih, W. Y.; Shih, W. H., "Effect of length, width, and mode on the mass detection sensitivity of piezoelectric unimorph cantilevers," Journal of Applied Physics, 91, 1680-1686, 2018.

[5] Lavrik, N. V.; Datskos, P. G., "Femtogram mass detection using photothermally actuated nanomechanical resonator". Applied Physics Letters, 82(16), 2697-2699, 2013.

[6] Ilic, B.; Czaplewski, D.; Zalalutdinov, M.; Craighead, H. G.; Neuzil, P.; Campagnolo, C.; Batt, C.," Single cell detection with micromechanical oscillators," Journal of Vacuum Science and Technology B: Microelectronics and Nanometer Structures, 19(6), 2825-2828, 2011.

[7] Carrascosa, L. G.; Moreno, M.; Alvarez, M.; Lechaga, L. M., "Nanomechanical biosensors: A new sensing tool," TrAC - Trends in Analytical Chemistry, 25 (3), 196-206, 2016.

[8] Raiteri, R.; Grattarola, M.; Butt, H. J.; Skladal, P, "Micromechanical cantilever-based biosensors. Sensors and Actuators", B: Chemical, 79 (2-3), 115-126, 2001.

[9] Allen, M. G. "Design, fabrication, and application of magnetic micro actuators. Micro Machine and Human Science," 5, 2017.

[10] Uzairue Stanley, Victor Matthews Olu, Charles Ochonogor, Amaize Peter, Anyasi Francis, "Experimental Analysis of Cable Distance Effect on Signal Attenuation in Single and Multimode Fiber Optics," International Journal of Electrical and Computer Engineering (IJECE), Vol.8, No.3, pp.1577 1582, June 2018.

[11] Budde T, G. H., "Thin film SMCO magnets for use in electromagnetic micro actuators," Journal of Applied Physics, 2006.

[12] Hisham Kadhum Hisham, (2018)., "Optical Fiber Sensing Technology: Basics, Classifications and Applications," American Journal of Remote Sensing; 6(1): 1-5 
[13] I Bouneb, F Kerrour, "Nanometric Modelization of Gas Structure, Multidimensional using COMSOL Software," International Journal of Electrical and Computer Engineering (IJECE) Vol.8, No.4, pp.2014 2020, August 2018.

[14] C, Z.," Cantilever-based biosensors. Analytical and Bioanalytical Chemistry," 946-959, 2014.

[15] D. de Bhail'1s, C. M,"Modelling and analysis of a magnetic micro actuator. Sensors and Actuators," 285-289, 2000.

[16] S. L. Biswal, "Micro and- nanocantilever systems for molecular analysis," in Nano and Microsensors for Chemical and Biological Terrorism Surveillance, J. B.-H. Tok, Ed., RSC Publishing, pp. 82-97, 2008.

[17] M. Yue et al., "A 2-D microcantilever array for multiplexed biomolecular analysis," Journal of Microelectromechanical Systems, vol. 13, pp. 290-299, 2004.

[18] Z.J. Davis, G. Abadal, B. Helbo, O. Hansen, F. Campabadal, F. Perez Murano, J. Esteve, E. Figueras, J. Verd, N., "Barniol, and A. Boisen. Monolithic integration of mass sensing nano-cantilevers with CMOS circuitry," Sensors and Actuators A: Physical, 105(3):311-319, 2013.

[19] F. Shen, P. Lu, S.J. O'Shea, K.H. Lee, and T.Y. Ng, "Thermal effects on coated resonant microcantilevers," Sensors and Actuators A: Physical, 95(1):17-23, 2017.

[20] P. Wang et al., "A micro electromagnetic low-level vibration energy harvester based on MEMS technology," Microsyst. Technol., vol. 15, no. 6, pp. 941-951, 2014.

[21] C. T. Pan et. al., "Fabrication and analysis of a magnetic self-power microgenerator," J. Mag. Magn. Mater., vol. 304, no. 1, pp. e394-e396, 2006.

[22] M. N. Saravana Kumar, R. Murugna, "Analysis of Inductance Gradient and Current Density Distribution Over Different Cross-section of Rails," International Journal of Electrical and Computer Engineering (IJECE), Vol. 8, No. 2, pp. 723 729, April 2018.

[23] Ergeneman O, E. P., "An in-plane cobalt-nickel micro resonator sensor with magnetic actuation and readout," Sensors and Actuators A: Physical, 1399-1408, 2017.

[24] Ajay V.G., Parvathy A.R., Thomaskutty Mathew, "Microstrip antenna with DGS based on CSRR array for WiMAX applications," International Journal of Electrical and Computer Engineering (IJECE), Vol. 9, No. 1, pp. 157 162, February 2019.

[25] Laure K. Lagorce, O. B.," Magnetic Micro Actuators Based on Polymer Magnets," IEEE Journal of Microelectromechanical Systems, 2-9, 2009.

[26] Tamayo J, H. A.,"Chemical sensors and biosensors in liquid environment based on microcantilevers with amplified quality factor," Ultramicroscopy, 167-173, 2018.

[27] Hisham K. Hisham, Ahmed F. Abas, Ghafoor A. Mahdiraji and Mohammed A. Mahdi, "Characterization of TurnOn Time Delay in a Fiber Grating Fabry-Perot Lasers”, IEEE Photonics Journal, 2013.

\section{Appendix}

A. The vertical electromagnetic force $\mathrm{F}_{\mathrm{z}}$ : acting on a magnet $\mathrm{M}_{\mathrm{z} \text { : }}$ of corrected by the demagnetization effect and volume $\mathrm{V}$ : from the equation;

$$
\mathrm{F}_{\mathrm{z}}=\mathrm{M}_{\mathrm{z}} \int_{\Delta \mathrm{V}} \frac{\mathrm{dH}_{\mathrm{z}}}{\mathrm{dz}} \mathrm{dV}
$$

B. The static deflection of a cantilever beam, subject to a concentrated load at its free end given as

$$
\delta=\frac{F L^{3}}{3 E I} \quad I=\frac{b h^{3}}{12}
$$

C. The relationship between the force and the deflection is given simply as:

$$
\mathrm{F}_{\mathrm{z}}=-\mathrm{kd}_{\mathrm{z}}
$$

$F$ : Force $(\mathrm{N}), \mathrm{d}$ : deflection in z-direction, k: the spring constant for a membrane material

D.

$$
\mathrm{d}_{\mathrm{z}}=\mathrm{c} \frac{\mathrm{F}_{\mathrm{z}} \mathrm{l}_{\mathrm{m}}}{\mathrm{D}}
$$

$\mathrm{d}_{\mathrm{z}}$ : is the deflection, $\mathrm{F}$ : is the applied force and D: is the flexural rigidity of the membrane material, $1_{\mathrm{m}}$ is the length of membrane and c: constant value depends on case. 